\title{
Indicadores da qualidade do solo em áreas cultivadas com goiaba
}

Vários são os perímetros irrigados no Nordeste brasileiro que apresentam problemas de degradação física e química dos solos resultantes da falta de informações sobre as potencialidades e limitações de uso, assim nos últimos anos é crescente a preocupação com o uso sustentável e a qualidade dos recursos naturais, especialmente do solo e da água, fazendo-se necessário, além de quesitos sociais e econômicos para expansão da agricultura, considerar também os aspectos relacionados à qualidade no manejo da água e do solo capazes de garantir a sustentabilidade dos sistemas de produção em longo prazo. Nesse sentido este trabalho propôs avaliar e comparar alterações químicas em áreas com diferentes tempos de cultivo com goiaba no Projeto de Irrigação do Baixo/Açu, RN, em relação à mata nativa da Caatinga. Para a área de referência, mata nativa de Caatinga, foram retiradas vinte amostras simples e em cada uma das cinco áreas cultivadas, uma amostra composta, formada a partir de vinte amostras simples. As características avaliadas foram cálcio, magnésio, potássio, sódio, fósforo, pH e CE e os metais tóxicos chumbo, níquel, cádmio, cobre, zinco, manganês e ferro; desta forma, observou-se melhoria da qualidade ambiental para $\mathrm{Ca}$, $\mathrm{Pb}$, $\mathrm{Ni}$, $\mathrm{Mn}$ e Fe das áreas cultivadas em relação à mata nativa e para os metais tóxicos os menores índices para $\mathrm{Cd}$, Cu e o Zn, indicaram alteração quando comparados com a mata nativa.

Palavras-chave: Manejo do solo; Índice de qualidade do solo; Cultivo agrícola.

\section{Soil quality indicators in guava orchards}

\begin{abstract}
Several are the irrigated perimeters in the Brazilian Northeast that present problems of physical and chemical degradation of the soils resulting from the lack of information about the potentialities and limitations of use, so in recent years the concern with the sustainable use and the quality of the natural resources has increased, especially of soil and water. In addition to social and economic considerations for the expansion of agriculture, it is also necessary to consider aspects related to water and soil quality that can guarantee the long-term sustainability of production systems. In this sense, this work proposed to evaluate and compare chemical changes in areas with different guava cultivation times in the Irrigation Project of Baixo-Açu, RN, in comparison with the native vegetation of the Caatinga. For the reference area, the native vegetation of the Caatinga, we took 20 simple samples and from each of the five guava orchards, we took a composite sample, which was composed of 20 simple samples. The evaluated features were calcium, magnesium, potassium, sodium, phosphorus, $\mathrm{pH}$, and EC, and the heavy metals lead, nickel, cadmium, copper, zinc, manganese, and iron. After the study, we noticed an improvement of the environmental quality for $\mathrm{Ca}, \mathrm{Pb}, \mathrm{Ni}, \mathrm{Mg}$, and $\mathrm{Fe}$ for the guava orchards in comparison with the native vegetation, and for the heavy metals, lower indexes of $\mathrm{Cd}, \mathrm{Cu}$, and $\mathrm{Zn}$ in comparison with the native vegetation indicated changes.
\end{abstract}

Keywords: Soil handling; Soil quality index; Agricultural cultivation.

Topic: Uso de Recursos Naturais

Reviewed anonymously in the process of blind peer
Received: $10 / 02 / 2019$

Approved: 25/03/2019
Elis Regina Costa Morais (ic

Universidade Federal Rural do Semi-Árido, Brasil

http://lattes.cnpq.br/1621529280559168

http://orcid.org/0000-0002-0545-0700

elisregina@ufersa.edu.br

Lívia Laiane Barbosa Alves

Universidade Federal Rural do Semi-Árido, Brasil

http://lattes.cnpq.br/6666812731337575

liviabarbosa17@yahoo.com.br

Mychelle Karla Teixeira de Oliveira (iD)

Universidade Federal Rural do Semi-Árido, Brasil

http://lattes.cnpq.br/0574275925807403

http://orcid.org/0000-0003-3264-5172

mymykar@gmail.com
Fernanda Medeiros Ramos

Universidade Federal Rural do Semi-Árido, Brasil

http://orcid.org/0000-0002-7497-7995

fernandarrmedeiros@outlook.com

Josinaide Cláudia Araújo de Santana (iD

Universidade Federal Rural do Semi-Árido, Brasil

http://lattes.cnpq.br/4791555972739746

http://orcid.org/0000-0001-8031-8729

josinaide1araujo@gmail.com

Jenickson Rayron da Silva Costa (iD

Universidade Federal Rural do Semi-Árido, Brasil

http://lattes.cnpq.br/7702395456210920

http://orcid.org/0000-0002-2321-6926

jenickson1@gmail.com
Referencing this:

MORAIS, E. R. C.; ALVES, L. L. B.; OLIVEIRA, M. K. T.; RAMOS, F. M.; SANTANA, J. C. A.; COSTA, J. R. S.. Indicadores da qualidade do solo em áreas cultivadas com goiaba. Revista Ibero Americana de Ciências Ambientais, v.10, n.2, p.331-339, 2019. DOI:

http://doi.org/10.6008/CBPC2179-6858.2019.002.0027 


\section{INTRODUÇÃO}

Com o crescimento da população mundial aumentou a necessidade de produzir mais alimentos, dessa forma, o manejo intensivo do solo, a monocultura, o uso dos pesticidas e fertilizantes tornaram-se práticas comuns para aumento da produção agrícola (OLIVEIRA et al., 2017), segundo o Programa das Nações Unidas para o Meio Ambiente através do GLSOD (Global Assessmentof Soil Degradation), o continente Sul Americano tem 244 milhões de hectares de solo degradado, no Brasil todas as estimativas apontam para o desmatamento, as atividades agrícolas e a mineração como as principais responsáveis pela degradação dos solos.

O desenvolvimento agrícola nos últimos anos promoveu aumento da produtividade, e para obter resultados positivos é necessária a união de diversos fatores, tendo o solo como principal recurso ambiental para o sucesso da produção agrícola, a fim de minimizar a sua degradação e adotar técnicas de manejo que contribuam para a manutenção ou recuperação da sua fertilidade. A qualidade do solo deve ser determinada para entender os limites que podem ser definidos para seu uso e tratamento (TRIPATHI et al., 2016), sendo essencial detectar precocemente mudanças na qualidade (TENG et al., 2014). Historicamente, para estabelecer a qualidade do solo, este que é um conceito relacionado às suas características intrínsecas e suas interações com o ecossistema e tipo de uso, utiliza-se atributos químicos, físicos e biológicos (KARLEN et al., 2001).

Atualmente, há um esforço multidisciplinar tentando quantificar diferentes atributos que estão relacionados com a sustentabilidade, traduzindo-os na forma de indicadores de qualidade do solo. Blanco et al. (2008) inferem que há algum grau de subjetividade quanto às percepções individuais do que pode ser considerado 'solo de boa qualidade'. Mcbratney et al. (2014) sugeriram levar em consideração a inclusão de conceitos mais complexos, como exemplo de 'segurança do solo', que trata da conservação e melhoria dos recursos do solo para produzir alimentos, fibra, água doce, energia e para ajudar a manter a sustentabilidade climática e biodiversidade, pois além de ter atributos biofísicos, inclui aspectos econômicos, sociais e políticos, originando assim um conceito de solo para o desenvolvimento sustentável, pois no cenário atual o aumento da demanda por alimentos isentos desses contaminantes, gerando assim mercados mais exigentes em relação à segurança alimentar (MARI et al., 2017).

O solo é um recurso natural fundamental, e sua qualidade é formada por fatores químicos, físicos, biológicos e ecológicos, podendo ser modificados em prol de melhor qualidade. A qualidade do solo é uma propriedade determinante para aumento de produtividade e sustentabilidade das culturas, influenciando também na saúde das plantas, animais e consequentemente seres humanos (MELO et al., 2017). O manejo inadequado e intensivo do solo pode ocasionar um estado de degradação que, caso seja reversível, requer muito mais tempo e recurso para sua recuperação (CHERUBIN et al., 2015). Assim, é necessário o monitoramento dos solos manejados para a preservação da sua qualidade e para que proporcione uma produção continuada. 
Embora a maioria dos autores avalie a qualidade do solo usando indicadores independentes, é preferível combinar alguns deles em modelos, a fim de criar um índice de qualidade do solo (IQS) que pode fornecer informações integradas sobre os processos e o funcionamento do solo (VAN LEEUWEN et al., 2015). Morais et al. (2014) estabeleceram índices de qualidade do solo é concluíram sob a importante na avaliação de impactos ambientais quando biomas são incorporados ao processo produtivo, seja de forma extensiva ou intensiva. Ainda segundo os autores, dentre os enfoques propostos para estabelecer critérios de referência, utiliza-se o solo de área com vegetação natural não alterada, visando representar as condições ecológicas ambientais e parâmetros agronômicos que maximizem a produção e conservem o meio ambiente.

Os indicadores de qualidade do solo são normalmente escolhidos de acordo com o foco da pesquisa, publicações recentes usam funções estatísticas para indicadores de grupo, com número inicial de parâmetros reduzido e escolhendo aqueles que melhor representam a qualidade do solo, podendo ser construídos de acordo com a opinião de especialistas (SÁNCHEZ-NAVARRO et al., 2015), com base na frequência os parâmetros aparecem em artigos científicos (ROUSSEAU et al., 2012), pode ser orientado unicamente sobre critérios estatísticos, e certamente, também pode consistir na combinação de ambas as estratégias (LIMA et al., 2013).

Nesse sentido, o nível de alteração na qualidade do solo pode ser avaliado pela mensuração do estado atual de determinados atributos em comparação com o estado natural do solo, sem interferência antrópica, ou com valores considerados ideais (SILVA et al., 2015), para Betini et al. (2014) o desafio é identificar os fatores ambientais que mais explicam a variação nas propriedades químicas. Dessa forma, objetivou-se avaliar a qualidade ambiental de áreas de cultivo de goiaba com diferentes tempos de uso, em relação à vegetação nativa.

\section{MATERIAIS E MÉTODOS}

O Projeto de Irrigação do Baixo Açu, está localizado no município do Alto do Rodrigues, no Estado do Rio Grande do Norte, esta região é conhecida pela prática da agricultura irrigada desde a década de 80 . De acordo com a classificação de Koppen essa área apresenta clima do tipo BSwh', ou seja, seco, muito quente e com estação chuvosa no verão atrasando-se para o outono, apresentando temperatura média anual de $27,4^{\circ} \mathrm{C}$, precipitação pluviométrica anual bastante irregular, com média de $673,9 \mathrm{~mm}$, e umidade relativa de $68,9 \%$ (CARMO FILHO et al., 1995).

Foram avaliadas cinco áreas, das quais, quatro correspondem a áreas com a cultura da goiaba (AC), sendo $A C 1, A C 2, A C 3$ e $A C 4$, com 4, 5, 6 e7 anos de cultivo, respectivamente, e outra é a área de referência/background (BG), sendo esta uma área de referência a mata nativa de Caatinga do entorno da cultura. A amostragem de solo foi feita de forma aleatória na camada de 0-20 cm, com auxílio de um trado holandês. $\mathrm{Na}$ área de referência/background (BG) as coletas foram realizadas aleatoriamente, visando reduzir a variabilidade do solo, coletando-se 60 amostras simples, as quais foram homogeneizadas, formando uma amostra composta. Quatro amostras compostas nas áreas de cultivo (AC) foram analisadas para estimar a média e o desvio padrão das características avaliadas. Após a coleta, os solos foram acondicionados em sacos 
plásticos identificados e levados ao laboratório e posteriormente analisados seguindo a metodologia recomendada pela EMBRAPA (2011).

As alterações da qualidade do solo das áreas de cultivo em relação à de referência, foram avaliadas pelo método proposto por Maia (2013), que leva em consideração a distribuição normal das características avaliadas e a comparação realizada com base nos desvios entre as áreas cultivadas e a de referência. Os dados de cada característica foram padronizados de acordo com a Eq. 1.

$$
\mathrm{Z}_{\mathrm{i}}=\frac{\mathrm{x}-\bar{x}}{\mathrm{~S}}
$$

em que: zi - valor padronizado da variável normal com média $\mu$ e desvio padrão $\sigma$ igual a zero e 1 , respectivamente $\mathrm{x}$ - valor da característica avaliada na área cultivada x e s - são a média e o desvio padrão da característica avaliada na área de referência, respectivamente. Para estimar os valores dos índices de qualidade ambiental de cada característica avaliada, utilizaramse as Eqs. 2, 3 e 4, para as condições de 'mais é melhor', 'menos é melhor' e 'valor máximo', respectivamente, $\operatorname{com} \beta=\exp (-1,7145$. zi), de acordo com Maia (2013), com IQi variando de 0 a 1 , sendo que quanto mais próximo a 1 melhor a qualidade do solo em relação à referência.

$$
\begin{aligned}
& \mathrm{IQ}_{\mathrm{i}}=\frac{1}{1+\beta} \\
& \mathrm{IQ}_{\mathrm{i}}=\frac{\beta}{1+\beta} \\
& \mathrm{IQ}_{\mathrm{i}}=\frac{4 \beta}{(1+\beta)^{2}}
\end{aligned}
$$

Das características avaliadas foram considerados como 'Quanto menos melhor' o sódio ( $\mathrm{Na}$ ), condutividade elétrica $(\mathrm{CE})$, chumbo $(\mathrm{Pb})$, níquel $(\mathrm{Ni})$ e o cádmio $(\mathrm{Cd})$ e, como 'valor máximo', potencial hidrogeniônico $(\mathrm{pH})$, fósforo $(\mathrm{P})$, cálcio $(\mathrm{Ca})$, magnésio $(\mathrm{Mg})$, potássio $(\mathrm{K})$, cobre $(\mathrm{Cu})$, zinco $(\mathrm{Zn})$, manganês ( $\mathrm{Mn}$ ) e ferro (Fe). Nenhuma das características avaliadas foi considerada como 'Quanto mais melhor'. O índice de qualidade do solo (IQS) foi calculado pela Eq. 5:

$$
I Q S=\frac{\sum_{i=n}^{n} I_{i}}{n}
$$

em que: IQS - índice de qualidade de solo da área avaliada IQi - índice de qualidade da característica avaliada n - número de características avaliadas

\section{RESULTADOS E DISCUSSÃO}

Verificou-se, dentre as características avaliadas, tendência de melhoria da qualidade ambiental para $\mathrm{Ca}, \mathrm{Pb}, \mathrm{Ni}, \mathrm{Mn}$ e $\mathrm{Fe}$; das áreas cultivadas em relação à mata nativa, enquanto para as para demais características, observou-se diminuição da qualidade ambiental (Tabelas 1 e 2). É necessário destacar que as maiores alterações foram para CE e P. As alterações referentes a $\mathrm{pH}$, provavelmente ocorre em razão da prática da irrigação, associadas ao baixo poder tampão dos solos com textura arenosa das áreas avaliadas, vindo a corroborar com Nunes et al. (2008). 
Tabela 1: Valores médios, desvio padrão e coeficiente de variação (CV) para área de background, valores da análise do solo das áreas cultivadas (AC) e índice de qualidade para características avaliadas.

\begin{tabular}{|c|c|c|c|c|c|c|c|}
\hline & $\mathrm{pH}$ & $\mathrm{CE}$ & $\mathrm{Ca}$ & $\mathrm{Mg}$ & $\mathrm{Na}$ & $\mathrm{K}$ & $\mathrm{P}$ \\
\hline & & $\mathrm{dS} \mathrm{m}^{-1}$ & \multicolumn{4}{|c|}{---------------------- $\mathrm{Cmol}_{\mathrm{C}} \mathrm{dm}^{-3}$----------------------- } & $\mathrm{mg} \mathrm{dm}^{-3}$ \\
\hline \multicolumn{8}{|c|}{------------------------------------------- Mata nativa ------------------------------------- } \\
\hline Média & 7,18 & 0,11 & 5,10 & 0,59 & 0,39 & 0,58 & 5,09 \\
\hline Desvio & 0,74 & 0,06 & 2,55 & 0,36 & 0,02 & 0,28 & 3,59 \\
\hline CV (\%) & 10,34 & 56,83 & 50,06 & 60,76 & 6,33 & 47,79 & 70,57 \\
\hline \multicolumn{8}{|c|}{ 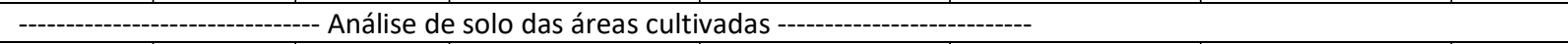 } \\
\hline AU1 & 7,70 & 0,32 & 3,80 & 0,84 & 0,73 & 1,88 & 19,18 \\
\hline AU2 & 7,47 & 0,86 & 0,00 & 0,00 & 0,81 & 0,70 & 26,52 \\
\hline AU3 & 7,37 & 0,16 & 4,88 & 1,20 & 0,47 & 0,53 & 14,37 \\
\hline AU4 & 7,91 & 0,16 & 4,52 & 1,12 & 0,56 & 0,54 & 42,73 \\
\hline \multicolumn{8}{|c|}{ - } \\
\hline AU1 & 0,714 & 0,003 & 0,831 & 0,722 & 0,000 & 0,105 & 0,005 \\
\hline AU2 & 0,898 & 0,000 & 0,122 & 0,212 & 0,000 & 0,105 & 0,000 \\
\hline AU3 & 0,955 & 0,221 & 0,995 & 0,200 & 0,003 & 0,105 & 0,047 \\
\hline AU4 & 0,530 & 0,198 & 0,963 & 0,279 & 0,000 & 0,105 & 0,000 \\
\hline
\end{tabular}

Tabela 2: Valores médios, desvio padrão e coeficiente de variação (CV) para área de background, valores da análise do solo das áreas cultivadas (AC) e índice de qualidade para características avaliadas.

\begin{tabular}{|c|c|c|c|c|c|c|c|}
\hline & $\mathrm{Pb}$ & $\mathrm{Ni}$ & $\mathrm{Cd}$ & $\mathrm{Cu}$ & $\mathrm{Zn}$ & $\mathrm{Mn}$ & $\mathrm{Fe}$ \\
\hline & \multicolumn{7}{|c|}{ 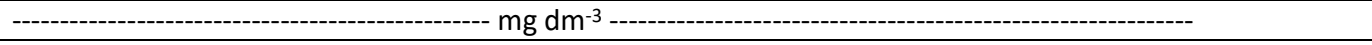 } \\
\hline \multicolumn{8}{|c|}{---or } \\
\hline Média & 2,20 & 1,09 & 0,18 & 0,31 & 2,69 & 33,93 & 1,44 \\
\hline Desvio & 0,29 & 0,44 & 0,04 & 0,10 & 1,40 & 32,13 & 1,77 \\
\hline CV (\%) & 13,01 & 40,12 & 22,51 & 31,86 & 51,96 & 94,70 & 122,92 \\
\hline \multicolumn{8}{|c|}{ 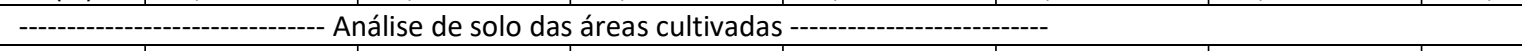 } \\
\hline AU1 & 0,00 & 0,01 & 1,59 & 2,34 & 3,49 & 2,59 & 0,00 \\
\hline AU2 & 0,00 & 0,54 & 1,08 & 1,94 & 2,73 & 0,00 & 0,91 \\
\hline AU3 & 0,81 & 1,44 & 2,68 & 2,14 & 2,15 & 2,20 & 0,65 \\
\hline AU4 & 1,26 & 0,56 & 2,99 & 4,36 & 8,51 & 0,60 & 0,00 \\
\hline \multicolumn{8}{|c|}{ 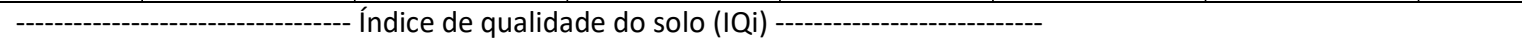 } \\
\hline AU1 & 1,000 & 0,986 & 0,000 & 0,000 & 0,797 & 0,533 & 0,637 \\
\hline AU2 & 1,000 & 0,896 & 0,000 & 0,000 & 1,000 & 0,483 & 0,936 \\
\hline AU3 & 1,000 & 0,201 & 0,000 & 0,000 & 0,000 & 0,525 & 0,868 \\
\hline AU4 & 0,996 & 0,887 & 0,000 & 0,000 & 0,003 & 0,495 & 0,637 \\
\hline
\end{tabular}

Nas áreas cultivadas os valores de pH variaram de 7,37 a 7,91 apresentando alcalinidade; entretanto, a mata nativa apresentou o mesmo comportamento, com valor de $\mathrm{pH}$ acima da neutralidade, $\mathrm{pH}$ médio de 7,18 , valor de baixa disponibilidade de alguns nutriente no solo, como o fósforo e micronutrientes, fato este também descrito por Gomes et al. (2006), os autores ainda concluem que solos ideais para cultivo devem apresentar pH entre 6,0 e 6,5. De acordo com Longo et al. (2005) a elevação do pH nas áreas cultivadas pode ser resultado da hidrólise de fertilizantes como, por exemplo, a ureia, muito utilizada pelos agricultores da área estudada.

Para a $\mathrm{CE}$, semelhante ao $\mathrm{pH}$, verificaram-se desvios positivos em todas as áreas (Tabela 1); entretanto, pode-se observar, em média, acréscimo de $235 \%$ quando comparadas as áreas de cultivo e a de mata nativa; isto representa uma variação expressa em valores médios de 0,016 a 0,086 dS m-1. Resultados similares foram encontrados por Chaves et al. (2006) que, analisando a salinidade e o impacto da irrigação no Perímetro Araras Norte, Ceará, observaram que o maior incremento de sais ocorreu na camada de 0 a 30 $\mathrm{cm}$, com uma CE de 2,16 dS $\mathrm{m}^{-1}$, enquanto que na mata nativa o valor equivalente foi de $0,32 \mathrm{dS} \mathrm{m}^{-1}$, representando, assim, um incremento de $577 \%$. Os autores enfatizaram que tal fato pode ser explicado pelo 
manejo da irrigação adotado pelo uso de fertilizantes minerais ricos em sais, como os cloretados e os nitrogenados.

Para todas as áreas avaliadas observou-se que os teores de Ca foram, em média, sempre maiores do que os de $\mathrm{Mg}$, com exceção da AU2, onde os teores de Ca e Mg foram iguais a zero (Tabela 1). Os maiores valores para o Ca ocorrem possivelmente pela série de retenção de cátions, que determina que o Ca é mais fortemente retido na matriz coloidal do solo do que o Mg (QUAGGIO, 2000). Os IQi do Mg foi menor que o do Ca, os IQi para Ca variaram de 0,122 a 0,995 (Tabela 3). Quanto aos menores desvios de K em relação ao $\mathrm{Ca}$ e $\mathrm{Mg}$, isto se deve, provavelmente, ao resultado da lixiviação deste elemento e devido às baixas concentrações de $\mathrm{K}^{+}$na água e ainda ao seu deslocamento do complexo sortivo dos solos provocado pelo $\mathrm{Ca}^{2+}$ e $\mathrm{Mg}^{2+}$ em concentrações muito mais elevadas na água (BRAUNER et al., 1982).

Tabela 3: Índice de qualidade do solo (IQS) para os diferentes números de ciclos das áreas cultivadas (AC)

\begin{tabular}{|l|l|}
\hline Áreas & IQS \\
\hline AC1 & 0,450 \\
\hline AC2 & 0,404 \\
\hline AC3 & 0,366 \\
\hline AC4 & 0,342 \\
\hline Coeficiente de correlação & $-0,9897$ \\
\hline
\end{tabular}

Com relação aos teores de sódio trocável $(\mathrm{Na})$, foram maiores nas áreas cultivadas com relação à área de referência, em média 65\%. Este acúmulo de Na está associado, possivelmente, à qualidade e ao manejo da água de irrigação (MORAIS et al., 2015). Assim, para não haver aumento de forma continuada no solo e futuramente favorecer problemas de produtividade nas áreas cultivadas, torna-se necessário um manejo efetivo da lâmina de irrigação utilizada (DANTAS et al., 2012). O Na apresentou os menores valores de IQi (Tabela 1).

Os teores, em média, de $\mathrm{P}$ na mata nativa foi de $5,09 \mathrm{mg} \mathrm{dm}^{-3}$ e variaram de 14,37 a $42,73 \mathrm{mg} \mathrm{dm}^{-3}$ nas áreas cultivadas (Tabela 1), observando-se o IQi para esta característica houve variações de acordo com o tempo de uso, onde o índice foi reduzido no maior tempo de cultivo (Tabela 3). Segundo Morais et al. (2014) o aumento do P nas áreas cultivadas pode ser explicado pelo efeito residual das adubações fosfatadas durante esses anos; no entanto, embora com as concentrações elevadas nessas áreas, é possível ocorrer a precipitação de $\mathrm{P}$ na forma de $\mathrm{Ca}_{3}\left(\mathrm{PO}_{4}\right)^{2}$ e adsorção ao $\mathrm{CaCO}_{3}$, em virtude do elevado $\mathrm{pH}$ e das altas concentrações de $\mathrm{Ca}^{2+}$ observadas no solo. Neste caso, como o extrator de P utilizado foi o Mehlich 1, que tem grande eficiência em recuperar $\mathrm{P}$ ligado ao cálcio, pode ocorrer superestimação da disponibilidade de $\mathrm{P}$, uma vez que esta forma de $\mathrm{P}$ não está prontamente disponível às plantas, à exceção daquelas que promovem acidificação em sua rizosfera, o que não ocorre com a cultura da bananeira (NUNES et al., 2008).

Para os metais tóxicos, destaca-se o alto valor do $\mathrm{Mn}, 33,93 \mathrm{mg} \mathrm{dm}^{-3}$, na área de mata nativa, aproximadamente 13 vezes maior que o valor mais alto encontrado para a AU1. Os teores de $\mathrm{Cd}, \mathrm{Cu}$ e $\mathrm{Zn}$ foram mais elevados nas áreas de cultivo quando comparados a área de vegetação nativa (Tabela 2), sendo o aumento mais expressivo para Cd, cujo aporte foi, em média, de 1035\%, em relação aos teores no solo com vegetação nativa e ainda os teores aumentaram com o tempo de cultivo (Tabela 2). O Cd, Cu e o $\mathrm{Zn}$ apresentaram valores de IQi muito baixos indicando alteração quando comparado com a Caatinga (Tabela 
2). A principal fonte desses elementos no solo são as adubações, principalmente a fosfatada, que provém de rochas com a presença desses metais.

Kassir et al. (2012) observaram, avaliando a mobilidade e a biodisponibilidade de $\mathrm{Cu}, \mathrm{Cd}, \mathrm{Zn}$ e Pb no solo adubados com fósforo durante 15 meses, que a sequência da mobilidade foi $\mathrm{Zn}>\mathrm{Cd}>\mathrm{Cu}>\mathrm{Pb}$ e aumento nas concentrações de $\mathrm{Cd}, \mathrm{Zn}, \mathrm{Pb}$ e $\mathrm{Cu}$ de 64, 26, 84 e 16\%, respectivamente, alertando para possível risco à cadeia alimentar e meio ambiente, sobremaneira as águas subterrâneas. Aumento nas concentrações de As, $\mathrm{Cd}$ e $\mathrm{Pb}$ em mais de 1000 áreas avaliadas na Califórnia, que receberam adubação fosfatada e com micronutrientes durante dezenas de anos foi verificado por Chen et al. (2008).

Levando em consideração as 14 características avaliadas neste trabalho e sabendo que a qualidade do solo é avaliada pelas características que influenciam no crescimento e desenvolvimento das plantas e ainda que o IQS considera a contribuição interativa de todos os atributos fornecendo uma avaliação abrangente da qualidade do solo, verificou-se, perdas expressivas de qualidade do solo nas áreas cultivadas, com relação à mata nativa, tendo coeficiente de correlação de -0,9897 (Tabela 3).

É importante salientar que os desvios das características estão sendo avaliados em relação à área de mata nativa, não significando que a mata nativa esteja na condição ótima para o cultivo agrícola. Teoricamente, o melhor IQS é igual a 1 (MAIA, 2013), para as condições estudadas, após quatro anos de cultivo, o IQS foi de 0,450, diminuindo ao longo dos anos até 0,342 , com sete anos de cultivo (Tabela 3); esses resultados corroboram com Maia (2013). Nesse sentido, Wienhold et al. (2004), concluíram que o índice de qualidade do solo estimou satisfatoriamente as alterações ocorridas no solo com os ciclos de cultivo em relação à mata nativa indicando que o uso desses índices é uma ferramenta apropriada para avaliar as alterações nos sistemas de cultivo; ressalta-se que esses autores utilizaram pesos para as características avaliadas.

\section{CONCLUSÕES}

Nas áreas cultivadas ocorreu decrescimento dos índices de qualidade ambiental com o tempo de cultivo quando relacionado à mata nativa; Observou-se melhoria da qualidade ambiental para $\mathrm{Ca}, \mathrm{Pb}, \mathrm{Ni}, \mathrm{Mn}$ e Fe e, para as demais características avaliadas, verificou-se diminuição nas áreas cultivadas em relação à mata nativa; Para os metais tóxicos, os menores índices para $\mathrm{Cd}, \mathrm{Cu}$ e o $\mathrm{Zn}$, indicam alteração quando comparados com a mata nativa.

\section{REFERÊNCIAS}

BERTINI, S. C. B.; AZEVEDO, L. C. B.; CARVALHO, I. C. M.; CARDOSO, E. J. B. N.. Hierarchical partitioning for selection of microbial and chemical indicators of soil quality. Pedobiologia, Camberra, v.57, n.4-6, p.293-301, 2014. DOI: https://doi.org/10.1016/j.pedobi.2014.06.001

BLANCO, H.; LAL, R.. Principles of Soil Conservation and Management. Springer: The Heidelberg, 2008.

BRAUNER, J. L.; GARCEZ, J. R. B.. Lixiviação de potássio, cálcio e magnésio em solos do Rio Grande do Sul submetidos à calagem, avaliada em condições de laboratório. Revista Brasileira de Ciência do Solo, Campinas, v.6, n.1, p.89-93, 1982.

CARMO FILHO, F.; OLIVEIRA, O. F.. Mossoró: Um município do semi-árido nordestino, caracterização climática e aspecto florístico. Mossoró: ESAM, 1995.

CHAVES, L. C. G.; ANDRADE, E. M.; CRISÓSTOMO, L. A.; NESS, R. L. L.; LOPEZ, J. F. B.. Risco de degradação em solo irrigado do Distrito de Irrigação do Perímetro Araras Norte, Ceará. 
Revista Ciência Agronômica, Fortaleza, v.37, n.3, p.292-298, 2006.

CHEN, W.; KRAGE, N.; WU, L.; PAN, G.; KHOSRIVAFARD, M.; CHANG, A. C.. Arsenic, cadmium, and lead in California cropland soils: Role of phosphate and micronutrient fertilizers. Journal Environmental Quality, Madison, v.37, n.2, p.689-695, 2008. DOI: http://doi.org/10.2134/jeq2007.0444

CHERUBIN, M. R.; EITELWEIN, M. T.; FABBRIS, C.; WEIRICH, S. W.; SILVA, R. F.; SILVA, V. R.; BASSO, C. J.. Qualidade física, química e biológica de um Latossolo com diferentes manejos e fertilizantes. Revista Brasileira Ciência do Solo, Viçosa, v.39, n.2, p.615-625. 2015. DOI:

http://doi.org/10.1590/01000683rbcs20140462

DANTAS, J. D. N.; OLIVEIRA, T. S.; MENDONÇA, E. S.; ASSIS, C. P.. Qualidade de solo sob diferentes usos e manejos no Perímetro Irrigado Jaguaribe/Apodi, CE. Revista Brasileira de Engenharia Agrícola e Ambiental, Campina Grande, v.16, n.1, p.18-26, 2012.

EMBRAPA. Empresa Brasileira de Pesquisa Agropecuária. Manual de análise químicas de solos. 2ed. Dados eletrônicos. Rio de Janeiro: Embrapa Solos, 2011.

GOMES, M. A. F.; FILIZOLA, H. F.. Indicadores físicos e químicos de qualidade de solo de interesse agrícola. Jaguariúna: Embrapa Meio Ambiente. 2006.

KARLEN, D. L.; ANDREWS, S. S.; DORAN, J. W.. Soil quality: Current concepts and applications. Advances in Agronomy, Australia, v.74, n.1, p.1-39, 2001. DOI: https://doi.org/10.1016/S0065-2113(01)74029-1

KASSIR, L. N.; DARWISH, T.; SHABAN, A.; OLIVIER, G.; OUAINI, N.. Mobility and bioavailability of selected trace elements in Mediterranean red soil amended with phosphate fertilizers: Experimental study. Geoderma, Amsterdã, v.189-190, p.357368, 2012. DOI:

https://doi.org/10.1016/i.geoderma.2012.05.017

LIMA, A. C. R.; BRUSSAARD, L.; TÓTOLA, M. R.; HOOGMOED, W. B.; GOEDE, R. G. M.. A functional evaluation of three indicators sets for assessing soil quality. Applied Soil Ecology, Vancouver, v.64, p.194-200, 2013. DOI: https://doi.org/10.1016/i.apsoil.2012.12.009

LONGO, R. M.; MELO, W. J.. Hidrólise da ureia em latossolos: Efeito da concentração de ureia, temperatura, $\mathrm{pH}$, armazenamento e tempo de incubação. Revista Brasileira de Ciência do Solo, Viçosa, v.29, n.4, p.651-657, 2005.

MAIA, C. E.. Qualidade ambiental em solo com diferentes ciclos de cultivo do meloeiro irrigado. Ciência Rural, Santa Maria, v.43, n.4, p.603-609, 2013.

MARI, C. L.; TAVARES, P. D. V. B.; FONSECA, V. M.. Alimentos, saberes e educação para o 'bem viver': os camponeses um passo adiante. Revista Eletrônica do Mestrado em Educação Ambiental, Rio Grande, v.34, n.3, p.37-54, 2017.

MCBRATNEY, A.; FIELD, D. J.; KOCH, A.. The dimensions of soil security. Geoderma, Amsterdan, v.213, p.203-213, 2014. DOI: https://doi.org/10.1016/j.geoderma.2013.08.013
MELO, V. F.; SILVA, D. T.; EVALD, A.; ROCHA, P. R. R.. Chemical and biological quality of the soil in different systems of use in the savanna environment. Revista Agro@mbiente on-line, Roraima, v.11, n.2, p.101-110, 2017. DOI: http://doi.org/10.18227/1982-8470ragro.v11i2.3850

MORAIS, E. R. C.; MAIA, C. E.; GAUDÊNCIO, HIARA R. S. C.; SOUSA, D. M. M.. Indicadores da qualidade química do solo em áreas cultivadas com mamoeiro irrigado. Revista Brasileira de Engenharia Agrícola e Ambiental, Campina Grande, v.19, n.6, p.587-591, 2015. DOI: http://dx.doi.org/10.1590/1807-1929/agriambi.v19n6p587$\underline{591}$

MORAIS, E. R. C.; OLIVEIRA, A. A. S.; MAIA, C. E.. Qualidade do solo cultivado com banana irrigada e sua relação com áreas de caatinga. Revista Brasileira de Engenharia Agrícola e Ambiental, Campina Grande, v.18, n.9, p.887-891, 2014. DOI: http://dx.doi.org/10.1590/18071929/agriambi.v18n09p887-891

NUNES, W. A. G. A.; KER, J. C.; NEVES, J. C. L.; RUIZ, H. A.; BEIRIGO, R. M.; BONCOMPANI, A. L. P.. Características químicas de solos da região de Janaúba, MG, irrigados com água de poços tubulares e do Rio Gorutuba. Revista Brasileira de Ciência do Solo, Viçosa, v.32, n.1, p.227-236, 2008.

OLIVEIRA, L. G.; BATALHA, M. O.; PETTAN, K. B.. Comparative assessment of the food purchase program and the national school feeding program's impact in Ubá, Minas Gerais, Brazil. Ciência Rural, Santa Maria, v.47, n.1, p.01-06, 2017. DOI: http://doi.org/10.1590/0103-8478cr20160395

QUAGGIO, J. A.. Acidez e calagem em solos tropicais. Campinas: Instituto Agronômico de Campinas, 2000.

ROUSSEAU, G. X.; DEHEUVELS, O.; RODRIGUES ARIAS, I.; SOMARRIBA, E.. Indicating soil quality in cacao-based agroforestry systems and old-growth forest: the potential of soil macrofauna assemblage. Ecological Indicators, Amsterdã, v.23, p.535-543, 2012. DOI: https://doi.org/10.1016/j.ecolind.2012.05.008

SÁNCHEZ-NAVARRO, A.; GIL-VÁSQUEZ, J. M.; DELGADOINIESTA, M. J.; MARÍN-SANLEANDRO, P.; BLANCOBERNARDEAU, A.; ORTIZ-SILLA, R.. Establishing an index and identification of limiting parameters of characterizing soil quality in Mediterranean ecosystems. Catena, Amsterdã, v.131, p.35-45, 2015. DOI:

https://doi.org/10.1016/j.catena.2015.02.023

SILVA, G. F.; SANTOS, D.; SILVA, A. P.; SOUZA, J. M..

Indicadores de qualidade do solo sob diferentes sistemas de uso na Mesorregião do Agreste Paraibano. Revista Caatinga, Mossoró, v.28, n.3, p.25-35, 2015. DOI: http://dx.doi.org/10.1590/1983-21252015v28n303rc

TENG, Y.; WU, J.; LU, S.; WANG, Y.; JIAO, X.; SONG, L.. Soil and soil environmental quality monitoring in China: $A$ review. Environment International, Kidlington, v.69, p.177199, 2014. DOI:

https://doi.org/10.1016/j.envint.2014.04.014 
TRIPATHI, R.; SHUKLA, A.K.; SHAHID, MD.; NAYAK, D.; PUREE, C.; MOHANTYA, S.; RAJA, R.; LAL, B.; GAUTAM, P. P.; BHATTACHARYY, B.B.; ANJANI, K.P.; JAMBHULKAR, N.N.; NAYAKE, A.K.. Soil quality in mangrove ecosystem deteriorates due to rice cultivation. Ecological Engineering, Kidlington, v.90, p.163-169, 2016. DOI:

https://doi.org/10.1016/j.ecoleng.2016.01.062

VAN LEEUWEN, J. P.; LEHTINEN, T.; LAIR, G. J.; BLOEM, J.; HEMERIK, L.; RAGNARSDÓTTIR, K. V.; GÍSLADÓTTIR, G.;
NEWTON, J. S.; RUITER, P. C.. An ecosystem approach to assess soil quality in organically and conventionally managed farms in Iceland and Austria. Soil, Berlim, v.1, p.83-101, 2015. DOI: http://doi.org/10.5194/soil-1-83-2015

WIENHOLD, B. J.; ANDREWS, S. S.; KARLEN, D. L.. Soil quality: A review of the science and experiences in the USA. Environmental Geochemistry and Health, Amsterdã, v.26, n.2, p.89-95, 2004. DOI: http://doi.org/10.1023/B:EGAH.0000039571.59640.3c

A CBPC - Companhia Brasileira de Produção Científica (CNPJ: 11.221.422/0001-03) detém os direitos materiais desta publicação. Os direitos referem-se à publicação do trabalho em qualquer parte do mundo, incluindo os direitos às renovaç̃ões, expansões e disseminações da contribuiç̃o, bem como outros direitos subsidiários. Todos os trabalhos publicados eletronicamente poderão posteriormente ser publicados em coletâneas impressas sob coordenação da Sustenere Publishing, da Companhia Brasileira de Produção Científica e seus parceiros autorizados. Os (as) autores (as) preservam os direitos autorais, mas não têm permissão para a publicação da contribuição em outro meio, impresso ou digital, em português ou em tradução. 